\title{
Implicit Learning of L2 Word Stress Rules
}

\author{
Ricky Chan (rickychan0809@gmail.com) \\ School of English, University of Hong Kong, Pokfulam Road, Hong Kong \\ Janny Leung (hiuchi@hku.hk) \\ School of English, University of Hong Kong, Pokfulam Road, Hong Kong
}

\begin{abstract}
This paper reports two experiments on the implicit learning of second language word stress rules and presents a methodological innovation. In both experiments L1 Cantonese L2 English participants practised pronouncing two-syllable Spanish words. Learning of a hidden stress regularity was measured by a judgment task. We assessed participants' awareness of the stress rule by verbal reports, confidence rating and a novel methodology: inclusionexclusion production tasks adapted from Destrebecqz and Cleeremans (2001). Experiment 1 demonstrated the implicit learning of association between the ending phoneme and word stress and experiment 2 the implicit learning of a more abstract rule of stress placement. We conclude that L2 word stress rules may be learnt implicitly.
\end{abstract}

Keywords: implicit learning, word stress, Spanish, binary confidence ratings, process dissociation procedure

\section{Introduction}

Previous studies of implicit learning have demonstrated that small-scale artificial grammar (Reber, 1967) and sequence of visual stimuli presentation in Serial Reaction Time (SRT) task may be learnt without awareness. In the domain of language, while early first language acquisition is essentially implicit, it remains cloudy whether implicit learning is relevant to second language acquisition (SLA) at all. The theoretical and pedagogical significance of implicit learning in SLA has stimulated recent research interests (e.g. Leung \& Williams, 2011; Robinson, 1996, 2005; Saffran, 2001; Saffran et al., 2008; Rebuschat \& Williams, 2011).

In the realm of L2 phonology, it has been shown that phonotactics (Dell, Reed, Adams, \& Meyer, 2000; Onishi, Chambers, \& Fisher, 2002; Warker \& Dell, 2006), segmental features (Goldrick, 2004) and metrical stress rules (Gerken, 2004; Gerken \& Bollt, 2008) may be learnt implicitly. Yet, to our knowledge, it is not known whether word stress regularities can be learnt without awareness, despite their importance in the parsing of speech stream. In the context of SLA, previous studies on the learning of lexical stress revealed that learners from a non-stress language background may have a different stress system than native speakers do, leading to a non-native "accent" of stress. For example, Mandarin speakers associated high flat tone with stress and Cantonese learners pronounce English stress and unstressed syllables as though they were high and low tones in their mother tongue (Chao, 1980). In light of the difficulty of learning stress, L2 stress pedagogy begs for further research. A crucial theoretical issue in implicit learning research is whether the knowledge acquired is abstract rules or memorised chunks. Knowlton and Squire $(1994,1996)$ have proposed that both abstract information about grammar patterns and concrete information about the learning materials may be involved in implicit learning and they both contribute to performance in grammaticality judgment tasks. Whether abstract rules or chunks are learnt is of great relevance to the learning of prosody in that it would shed lights on how knowledge of prosodic signals is represented.

Relevant studies have been met with methodological challenges. Critics have challenged the validity of assessing awareness by verbal reports. The inability to verbalise knowledge might reflect low confidence on the part of the participants, lack of appropriate vocabulary to describe their knowledge or the intrinsic complexities of the regularity, and the knowledge assessed by verbal reports might not be responsible for performance on the measure of learning (Dienes \& Berry, 1997; Shanks \& St. John, 1994). Dienes et al. (1995) have sought to improve awareness measurement by asking participants to rate their confidence in judgment; a lack of correlation between judgment performance and confidence rating suggests that knowledge is unconscious (zero-correlation criterion). Tunney and Shanks (2003) found that binary confidence ratings are more sensitive to low levels of awareness than continuous ratings and were therefore adopted in our experiment.

More recently, Destrebecqz \& Cleeremans (2001) have developed an objective awareness measure called the "method of opposition", modelled on the process dissociation procedure, a methodology framework first proposed by Jacoby (1991) to differentiate between the influences of implicit and explicit knowledge on performance. The method of opposition was applied to the SRT task (e.g. Curran, 2001; Haider, Eichler, \& Lange, 2010). After completing the SRT task, participants were told there was a hidden sequence governing the presentation of visual stimuli and they were asked to complete free-generation tasks under both inclusion and exclusion instructions. Participants were asked to press response keys in an order that followed the sequence (inclusion condition) and that did not resemble the sequence (exclusion condition). According to the Global Workspace theory (Baars, 2003), when knowledge becomes conscious, the possibility for voluntary control of performance is opened up. A difference between inclusion and the exclusion performance indicates top-down processing and thus explicit knowledge. On the contrary, people with no explicit knowledge would tend to perform equally well in both inclusion and exclusion tasks (inclusion = exclusion) as they do not have control over how the implicitly learnt knowledge 
influences behaviour (Curran, 2001). The experiment below demonstrates how the method of opposition could be employed as an awareness measure in other implicit learning tasks.

\section{Method}

\section{Experiment 1}

Materials The learning target was a simplified Spanish stress rule: words that end in " $O$ " have their stress on the penultimate syllable (e.g. busco and burro) and words that end in "ar" on the last syllable (e,g, gustar and tumbar). Two-syllable real Spanish words were employed as stimuli and they were generated, using a Spanish male diphone database $_{-}^{11}$, by the MBROLI speech synthesizer (Dutoit, Pagel, Pierret, Bataille, \& Vrecken, 1996). The specific values were based on those used by Enríquez, Casado and Santos (1989): $100 \mathrm{~Hz}$ and $60 \mathrm{~ms}$ for unstressed vowels and $116 \mathrm{~Hz}$ and $120 \mathrm{~ms}$ for stressed vowels. Albeit the artificiality of the synthesizer, it was still preferred to voice recordings because a Spanish native speaker is likely to be biased against incorrectly stressed words, which raises a possibility that participants rely on the speaker's fluency rather than knowledge of stress placement in the judgement task.

Procedure 37 university students (14 males and 16 females, Mage $=21.8$ years old) were recruited as the experimental group and 15 university students aged 20 to 26 (7 males and and 8 females, Mage 21.4) as the control group. All of them were native Cantonese speakers with English as an L2. None of them reported any knowledge of Spanish or Portuguese. They were told that the experiment aimed to study how people learn words. The experiment took around 25 minutes to complete.

Training phase: The training phase consists of 64 randomised trials, each containing a Spanish word and its English translation (See Fig 1, left). A set of 16 Spanish words, half of which end with -ar and the other half $-o$, was repeated four times. Participants repeated aloud after the recording. This provided participants with exposure to the target stress rules without explicitly directing their attention to them.

Testing Phase (pronunciation judgement): The testing phase consists of 40 trials, each of which includes an English verb and two sound icons (See Fig 1, right). Participants pressed the corresponding keys to listen to two audio presentations and choose the one that "sounds better" to them. As Scott and Dienes (2008) have shown that familiarity is the essential source of knowledge in artificial grammar learning, preference judgment is used rather than accuracy judgment (e.g., "choose the correct one") because the former may encourage the use of intuition and discourage rule search during the testing phase (Rebuschat \& Williams, 2011).

\begin{tabular}{l|ll} 
speak & \multicolumn{2}{|c}{ like } \\
hablar & Word 1 & Word 2
\end{tabular}

Figure 1: A sample trial used in learning (left) and testing phase (right) in experiment 1

All the 16 critical items (8 -ar ending and $8-o$ ending) were novel to the participants. Those sound pairs differed only in stress placement. Other previously seen items were randomly shuffled with the critical items so as to reduce the likelihood of participants consciously deducing the difference between the sound pairs.

To test whether, for -ar ending words, participants' judgements were based on the $-a r$ ending, $-r$ ending or the vowel " $a$ " at the end, $8-a$ ending words were added in the testing phase. Like the -0 ending words, words that end in " $a$ " have their stress on the penultimate syllable and those sound pairs differed only in stress placement.

Inclusion-exclusion Tasks. Adapted from Destrebecqz and Cleeremans (2001), the tasks required participants to read aloud 8 two-syllable words in each of the two conditions: 1) "as similarly to Spanish pronunciation as possible" (inclusion) and 2) "as differently from Spanish pronunciation as possible" (exclusion). A small dot was given in each word (see Fig 2) to indicate syllabification which serves to remind the participants that all words consist of two syllables. Their voices were recorded.

\section{Tum.bar}

Figure 2: A sample trial in the inclusion-exclusion tasks

Verbal reports. Participants were probed for whether they had any knowledge about the pronunciation patterns and stress rules of Spanish words. They were also told there were underlying stress patterns and if they could not report knowledge of the regularities, they would be asked to provide as many guesses as possible.

All the items used are presented in table 1 below.

\footnotetext{
${ }^{1}$ http://tcts.fpms.ac.be/synthesis/mbrola.html
} 


\begin{tabular}{|c|c|c|c|}
\hline \multicolumn{4}{|c|}{ A: Items used in training (16 items) } \\
\hline \multicolumn{2}{|c|}{ Words ended in "ar" } & \multicolumn{2}{|c|}{ Words ended in "o" } \\
\hline Hablar & Barrar & Soplo & Borro \\
\hline Rascar & Roncar & Marco & Busco \\
\hline Tumbar & Lanzar & Zumbo & Forzo \\
\hline Contar & Gustar & Tosto & Gasto \\
\hline \multicolumn{4}{|c|}{ B: Critical items used in the testing phase (16 items) } \\
\hline \multicolumn{2}{|c|}{ Words ended in vowels } & \multicolumn{2}{|c|}{$\begin{array}{c}\text { Words ended in } \\
\text { consonants }\end{array}$} \\
\hline Probar & Tratar & Broto & Grabo \\
\hline Juzgar & Firmar & Colgo & Formo \\
\hline Saltar & Cantar & Bastar & Pinto \\
\hline Montar & Costar & Falto & Junto \\
\hline \multicolumn{4}{|c|}{ C: $-a$ ending words used in the testing phase ( 8 items) } \\
\hline Roba & Bota & Cita & Dota \\
\hline Mata & Vota & Paga & Toma \\
\hline \multicolumn{4}{|c|}{ D: Items used in inclusion-exclusion tasks (16 items) } \\
\hline \multicolumn{2}{|c|}{ Inclusion } & \multicolumn{2}{|c|}{ Exclusion } \\
\hline Ha.llo & Lle.nar & Lla.mar & Ce.rro \\
\hline Lle.vo & Cho.car & Ten.tar & Llo.ro \\
\hline Tum.bar & Bre.go & Chu.par & Pen.so \\
\hline Mando & Tar.dar & Car.go & Cor.tar \\
\hline
\end{tabular}

Table 1: Items used in training, testing, and inclusionexclusion tasks in experiment 1

\section{Results and Discussion}

Overall performance Participants' performance on the pronunciation judgment task served as the measure of learning. The experimental group attained an average of 59.6 $\%$ accuracy (SD $=1.41$; SEM $=.23$ ) on the 16 critical items. Further analysis using $t$-test showed that their performance was significantly above chance, $t(36)=6.57, p<<.001, d=$ 1.55. They achieved above-chance-level performances on both - ar ending and -0 ending words, $M=64.2 \%$, SD $=1.42$, $\mathrm{SEM}=.24, t(36)=4.81, p<<.001, d=1.13$ and $M=55.1 \%$, $\mathrm{SD}=1.13, \mathrm{SEM}=.19, t(36)=2.16, p=.019, d=.51$ respectively. Their performance on $-a r$ ending words was significantly better than that of $-o$ ending words, $t(69)=2.41$, $p<0.01, d=.57$, suggesting more learning of - ar ending words than $-O$ ending words in relation to stress placement.

Awareness measures Verbal reports and inclusion-exclusion tasks were used to determine whether the acquired knowledge was conscious or not.

Verbal reports: 32 out of 37 participants remained unaware of the underlying stress rules based on verbal reports. One participant was able to verbalize the whole target stress rules.
Four participants reported some knowledge that overlaps with our target rules (i.e. " $r$ " is stressed; intonation falls when a words ends in $-O$; stress is related to the "ar" ending; stress is related to word length). They were classified as aware. In fact many participants did not notice any stress patterns and were surprised when they were asked to guess.

Inclusion-exclusion tasks: Participants' audio recordings for the tasks were analysed using Praat to locate their stress placement based on the fundamental frequencies (F0) of the two syllables, as it was found that Chinese speakers rely on F0 as the most important cue in stress judgement (Wang, 2008). The difference between inclusion and exclusion scores was calculated. The five aware participants scored higher under the inclusion instruction than the exclusion instruction (from +2 to +3 ), showing congruence with their awareness level revealed in verbal reports. 15 out of the 32 remaining participants scored equally for both tasks and they were classified as our truly unaware participants. The other 17 participants who showed some difference in their scores for both tasks (from -3 to +3 ) were re-classified as aware and were not included in our analysis of unaware data.

Pronunciation Judgment Task: The 15 unaware participants achieved an average of $58.8 \%$ accuracy $(M=9.4 ; S D=1.20$; $S E M=.32$ ) on the 16 critical items and their performance was significantly above chance, $t(14)=4.37, p<<.01, d=$ 1.65. Their performances on both - ar ending and -0 ending words are both significantly above chance, $M=59.1 \%, S D=$ $1.12, S E M=.30, t(14)=2.44, p=.014, d=.92$ and $M$ $=58.3 \%, S D=1.07, S E M=.29, t(14)=2.32, p=.018, d=$. 88 respectively. There is evidence of implicit learning of L2 ending-phoneme-to-stress rules by young Cantonesespeaking adults with only short and limited exposure. However, their accuracy on -ar ending words was not significantly higher than that of $-O$ ending words, $t(28)=$ $0.16, p=0.44$.

Control: The control group completed only the pronunciation judgement task. Their accuracy on critical items was not significantly different from chance, $M=47.1 \%, \mathrm{SD}=1.09$, $\mathrm{SEM}=.29, t(14)=1.61, p=.065$.

While previous studies suggest that novel L2 stress perception may pose challenges to learners from a non-stress language background, participants' proficiency in English and their prior general knowledge of word stress might have been helpful in this experiment. Moreover, it was found that participants in general performed significantly better for -ar ending words than $-O$ ending words. This may be explained by their prior linguistic knowledge. A previous study (Bailey, Plunkett, \& Scarpe, 1999) showed that native English speakers, when learning a novel stress pattern, had a significant bias for non-word-final stress. In our study, when participants were asked if they had any feelings about pronunciation patterns of Spanish words, 12 participants mentioned "the intonation of the words tended to go up", "the last syllable seemed to be louder and higher in pitch" and "stress tends to lie on the final syllable", despite the fact that words with a word-final stress and a non-word-final stress appeared equally frequently in the experiment. These 
statements are an indication that stress patterns that are distinct from English (word-final stress in this case) might have appeared more salient to our participants given their prior linguistic experience. In addition, heavy syllables tend to stressed in English. This may explain why there appeared to be more learning of -ar ending words (which has a heavy syllable at the end) than that of $-O$ ending words.

It is worth mentioning that there is a medium negative correlation between participants' accuracy on -ar ending words and that of $-a$ ending words, $r(35)=-0.51, p=.001$ for all participants and $r(13)=-0.72, p=.002$ for unaware participants. On the other hand, a medium positive correlation was found between their accuracy on $-O$ ending words and that of $-a$ ending words for both the whole experimental group, $r(35)=0.49, p=.002$, and the unaware participants, $r(13)=0.64, p=.001$. These suggest that participants might have made their judgements based on the last phoneme (i.e. the $-r$ ending instead of the vowel $a$ ) in the last syllable. While it is still unclear whether participants learnt an abstract rule or a set of probabilities, their higher sensitivity to the difference between open and closed syllables provided initial evidence of rule learning. This issue was addressed in a follow-up experiment, whose design was similar to experiment 1.

\section{Method}

\section{Experiment 2}

Materials The learning target was more complicated stress regularities: words that end in a consonant (closed syllable) have their stress on the final syllable (e.g. felol and cerroz) and words that end in a vowel on the last syllable (e,g, pato and bona). Two-syllable nonwords with four or five phonemes (vowel-ending words and consonant-ending words respectively) were generated by the same speech synthesizer based on Spanish pronunciation. The words were combination of phonemes illustrated below:

1. First phoneme: /b/, /b/, /d/, /d/, /f/, /g/, /h/, /k/, /k/, / / (ll), /m/, /n/, /p/, /s/, /s/, /t/, /t/ or /v/.

2: Second phoneme: /a/, /e/ or $/ \mathrm{o} /$.

3. Third phoneme: /b/, /b/, /d/, /d/, /g/, /k/, /k/, /l/, /m/, /n/, /n/, /p/, / /, /r/ (trilled), /s/, /t/, /t/ or /v/.

4. Fourth phoneme: /a/, /e/ or /o/

5. Fifth phoneme (consonant-ending words only): / /, /l/, / $\theta$ / (z).

Procedure 44 participants were recruited. The experimental group consisted of 22 young adults (9 male and 13 female, Mage $=21.0$ years old). The other 22 young adults (12 male and 10 female, Mage = 22.3 years old) were the control group. They were all native Cantonese speakers with English as an L2 with no knowledge of Spanish or Portuguese. The experiment took around 30 minutes to complete.

Training phase: Participants were presented with a Spanish word (See Fig 2, left). They repeated aloud after the recording. 36 Spanish-based words, half of which end in a vowel and the other half consonant, were repeated three times to form 108 trials.

Testing Phase (pronunciation judgement): Participants clicked to listen to two possible Spanish pronunciations (See Figure 2, right) and chose the one that "sounds better" to them.

\section{domal Word $1 \quad$ Word 2}

\section{Figure 2: A sample trial used in learning (left) and testing} phase (right) in experiment 2

Confidence rating: A binary confidence rating was included to further improve awareness assessment. After making a judgement, participants were asked to indicate whether they made a guess or were certain about their choice.

Critical data came from 18 novel words, half of which ends in /a/, /e/ or /o/ and the other half / $/$ /, /l/ and /z/. To determine whether participants had learnt an abstract rule or memorised chunks, 12 “extension items”, half of which ends in /i/ or /u/ (vowel ending) and the other half /d/, / x/ (consonant ending) were included. Those sound pairs differed only in placement of stress.

Inclusion-exclusion tasks: Same as experiment 1, except that there were 18 items for each condition.

Verbal reports: Same as experiment 1.

\begin{tabular}{|c|c|c|c|c|c|}
\hline \multicolumn{6}{|c|}{ A: Items used in training ( 36 items) } \\
\hline - a ending & -o ending & -e ending & -r ending & -lending & - $z$ ending \\
\hline Beba & Gobo & Coge & Botor & Bogel & Cerroz \\
\hline Bona & Navo & Dade & Coder & Domal & Gapez \\
\hline Cepa & Pato & Fane & Llaner & Debal & Hocaz \\
\hline Doca & Seco & Mete & Penar & Felol & Natoz \\
\hline Hara & Sorro & Tome & Socor & Madel & Tobaz \\
\hline Llada & Telo & Vese & Tevar & Sasol & Verez \\
\hline \multicolumn{6}{|c|}{ B: Critical items used in the testing phase (18 items) } \\
\hline -a ending & -o ending & -e ending & -r ending & -lending & -z ending \\
\hline Dada & Goto & Cebe & Decar & Mebel & Cepez \\
\hline Moda & Llemo & Sage & Llager & Savol & Gadoz \\
\hline Teca & Savo & Tope & Tomor & Sotal & Todaz \\
\hline \multicolumn{6}{|c|}{ C: Extension items used in the testing phase (12 items) } \\
\hline \multicolumn{2}{|c|}{-i ending } & -u ending & \multicolumn{2}{|c|}{-d ending } & $\mathrm{j}$ ending \\
\hline \multicolumn{2}{|c|}{ Llepi } & Dotu & \multicolumn{2}{|c|}{ Saded } & Gotej \\
\hline \multirow{2}{*}{\multicolumn{2}{|c|}{ Gomi }} & Sacu & Cemo & & Llecaj \\
\hline & & Tedu & Tobac & & Dapoj \\
\hline D: I & tems used & in inclusion & $\mathbf{x c l u s i o n} \mathbf{t}$ & sks (18 ite & ms) \\
\hline -a ending & -o ending & -e ending & -r ending & -l ending & -z ending \\
\hline Ho.na & So.to & Ta.re & To.bar & Vo.sal & De.rraz \\
\hline Ce.ba & Ga.lo & Lle.de & Ca.mer & Ba.pel & Fo.gez \\
\hline No.ca & Ba.vo & Me.te & Se.nor & Pe.col & Da.doz \\
\hline Ga.ba & Me.no & Va.de & Bo.ver & Ne.bol & $\mathrm{Da} \cdot \operatorname{coz}$ \\
\hline Te.la & Co.po & Se.ge & Cerror & Fo.tal & Te.maz \\
\hline Do.sa & Be.to & So.ne & Lle.car & Pa.rrel & Ho.dez \\
\hline
\end{tabular}

Table 2: Items used in training, testing, and inclusionexclusion tasks in experiment 2 


\section{Results and Discussion}

Verbal reports: Among the 22 participants, one participant was able to report our target rules. Two other participants mentioned "rising intonation for -z ending words" and "stress is affected by length of the word", which overlap with our target rules. These three participants were classified as aware. Still, many participants reported they paid no attention to any stress patterns and were surprised when they were asked to guess.

Confidence rating: The Chan difference score (Chan, 1992; Dienes et al, 1995) was computed for each participant to determine whether learning was implicit by the zero correlation criterion. It is the difference between the proportion of 'certain' responses which were correct (hit) and those which were incorrect (false alarm). Participants with a positive score were classified as aware and those score 0 or below were classified as unaware.

Inclusion-exclusion tasks: Participants who scored the same in inclusion and exclusion tasks were classified as unaware; otherwise they were classified as aware.

Pronunciation Judgment Task: Only 8 out of 22 participants were classified as unaware based on the above criteria. Their average accuracy in the judgement task was $68.1 \%(M=$ $12.25 ; S D=1.67 ; S E M=.59$ ) on the 18 critical items, which was significantly above chance, $t(7)=5.51, p<.001, d=$ 1.95. They achieved better-than-chance accuracy for both vowel and consonant ending words, $t(7)=2.12, p=.03, d=$ 0.75 and $t(7)=3.86, p=.003, d=1.37$ respectively, but accuracies on vowel and consonant ending words are not significantly different, $t(14)=.30, p=.39$. These serve as evidence of learning the target regularities.

An analysis of their accuracy on extension items showed that their accuracy was significantly higher than chance, $t(7)=$ 4.73, $p=0.001, d=1.67$. They achieved better-than-chance accuracy for both vowel and consonant ending words, $t(7)=$ $5.00, p<.0001, d=1.77$ and $t(7)=2.39, p=.023, d=0.85$ respectively, with no significant difference on accuracies for vowel and consonant ending words, $t(14)=1.25, p=.15$. Such findings revealed that the resultant knowledge was abstract rules rather than memorized chunks. Participants were sensitive to the difference between the ending phoneme (vowel or consonant) and syllable type (open and closed syllables) in relation to stress placement and were able to apply their abstract knowledge to novel items.

Control: The control group completed only the testing phase. Their accuracy on critical items was not significantly higher than chance, $M=52.0 \%, \mathrm{SD}=1.24, \mathrm{SEM}=.27 t(21)=.74$, $p=2.33$.

\section{Conclusion}

The two experiments above suggest that, under incidental learning conditions, young adults were able to learn L2 word stress rules, which are supra-segmental phonological patterns, with only short and limited exposure. The knowledge obtained was implicit, abstract, and may be applied to novel items. Their lack of awareness was verified by verbal reports, binary confidence ratings and process dissociation procedure in the experiments. We conclude that young adults are able to acquire L2 word stress implicitly.

The present study bears theoretical, methodological and pedagogical significance. On the theoretical level, it extends previous findings on the implicit learning of language: not only may syllable regularity (at the segmental level) and metrical stress rules be learnt implicitly (as shown in previous studies), but sensitivity to lexical stress rules (at the supra-segmental level) may also develop without awareness. This raises the possibility of implicit learning of other kinds of prosodic rules such as tonal rules. Importantly, the rules and stimuli in the experiment were all based on a natural language and so there is little question of transferability of findings to the context of language acquisition.

Our study is also methodologically interesting as it is the first implicit learning study which integrates verbal reports, subjective measures of confidence and process dissociation procedure to establish strict criteria for awareness measurement. It is also, to our knowledge, one of the first successful attempts to apply the process dissociation procedure outside of SRT tasks. Based on our data, the verbal reports were useful in identifying three aware participants who were able to verbalize partial knowledge of the target rules. However, other participants whose awareness was not reflected in the verbal reports nevertheless performed differently in the inclusion-exclusion tasks, which appeared to be more sensitive in identifying participants with low confidence or low awareness about the rules. The findings demonstrated the usefulness of inclusion-exclusion tasks as an objective measure of participants' awareness, and their potentially higher sensitivity than verbal reports.

From the perspective of L2 pedagogy, the present study provides an insight into how L2 stress patterns may be taught and learnt. The possibility of learning L2 stress patterns implicitly offers an alternative to the widely-adopted explicit approach to teaching word stress. It would be theoretically and practically interesting to determine the relative effectiveness of explicit and implicit teaching and learning of word stress and explore their potential synergetic effects in different settings.

Other further research directions include exploring 1) the implicit learning of other supra-segmental phonological rules such as tonal rules; 2) whether the ability to implicitly learn L2 phonological rules is sensitive to individual differences; and 3) whether age has a significant impact on the implicit learning of L2 phonological patterns.

\section{References}

Baars, B. J. (2003). How brain reveals mind: Neuoimaging supports the central role of conscious experience. Journal of Consciousness Studies, 10(9-10), 100-114.

Bailey, T., Plunkett, K., \& Scarpe, E. (1999). A crosslinguistic study in learning prosodic rhythms: Rules, constraints, and similarity. Language and Speech, 42(1), 138. 
Chan (1992). Implicit cognitive process: Theoretical issues and applications in computer systems design. Unpublished DPhil thesis, University of Oxford.

Chao, Y.R. (1980). Chinese tone and English stress. In Waugh, L. R. \& VanSchooneveld, C. H. (eds.) The Melody of Language, Baltimore, MD: University Park Press, 41-44

Curran, T. (2001). Implicit learning revealed by the method of opposition. Trends in Cognitive Sciences, 5, 503-504.

Dell, G. S., Reed, K. D., Adams, D. R., \& Meyer, A. S. (2000). Speech errors, phonotactic constraints, and implicit learning: A study of the role of experience in language production. Journal of Experimental Psychology: Learning Memory, and Cognition, 26(6), 1355-1367.

Destrebecqz, A., \& Cleeremans, A. (2001). Can sequence learning be implicit? New evidence with the process dissociation procedure. Psychonomic Bulletin \& Review, 8(2), 343-350.

Dienes, Z., Altmann, G.T.M., Kwan, L. \& Goode, A. (1995). Unconscious knowledge of articifial grammars is applied strategically. Journal of Experimental Psychology: Learning, Memory and Cognition, 21, 1322-1338.

Dienes, Z., \& Berry, D. C. (1997). Implicit learning: Below the subjective threshold. Psychonomic Bulletin and Review, 4, 3-23.

Dutoit, T., Pagel, N., Pierret, N., Bataille, O., \& Vrecken, V. d. (1996). The MBROLA project: Towards a set of highquality speech synthesizers free of use for non-commercial purposes. Proceedings of ICSLP'96, Philadelphia, 3(13931396).

Enríquez, E. V., Casado, C., \& Santos, A. (1989). La percepción del acento en español. Lingüística Española Actual, 11, 241-269.

Gerken, L. A. (2004). Nine-month-olds extract structural principles required for natural language. Cognition, 93, B89-B96.

Gerken, L. A., \& Bollt, A. (2008). Three examples allow at least some linguistic generalizations: Implications for generalization mechanisms and constraints. Language Learning and Development, 4, 228-248.

Goldrick, M. (2004). Phonological featurs and phonotactic constraints in speech production. Journal of Memory \& Language, 51, 586-603.

Haider, H., Eichler, A., \& Lange, T. (2010). An old problem: How can we distinguish between conscious and unconscious knowledge acquired in an implicit learning task? Consciousness and Cognition.

Jacoby, L. L. (1991). A process dissociation framework: Separating automatic from intentional uses of memory. Journal of Memory \& Language, 30, 513-541.

Juffs, A. (1990). Tone, syllable structure and interlanguage phonology: Chinese learners’ stress errors. International Review of Applied Linguistics, 28(2), 99-118.

Knowlton, B. J., Squire, L. R. (1994). The information acquired during artificial grammar learning. Journal of Experimental Psychology: Learning, Memory and Cognition, 20, 79-91.
Knowlton, B. J., Squire, L. R. (1996). Artificial grammar learning depends on implicit acquisition of both abstract and exemplar-specific information. Journal of Experimental Psychology: Learning, Memory and Cognition, 22, 169-181.

Leung, J., \& Williams, J. N. (2011). The Implicit Learning of Mappings between Forms and Contextually-Derived Meanings. Studies in Second Language Acquisition, 33.

Onishi, K., Chambers, K., \& Fisher, C. (2002). Learning phonotactic constraints from brief auditory experience. Cognition, 83, B13-B23.

N.Warner (eds.). Berlin: Mouton de Gruyter, 203-240.Reber, A. S. (1967). Implicit learning of artificial grammars. Journal of Verbal Learning and Verbal Behaviour, 6, 855863.

Rebuschat, P., \& William, J. N. (2011). Implicit and explicit knowledge in second language acquisition. Applied Psycholinguistics, Available on CJO 2011 doi:10.1017/S0142716411000580

Robinson, P. (1996). Learning simple and complex second language rules under implicit, incidental, rule-search, and instructed conditions. Studies in Second Language Acquisition, 18, 27-67.

Robinson, P. (2005). Cognitive abilities, chunk-strength, and frequency effects in implicit artificial grammar and incidental L2 learning: Replications of Reber, Walkenfeld, \& Hernstadt (1991) and Knowlton \& Squire (1996) and their relevance for SLA. Studies in Second Language Acquisition, 27, 235-268.

Saffran, J. R. (2001). The use of predictive dependencies in language learning. Journal of Memory and Language, 44(493-515).

Saffran, J. R., Hauser, M., Seibel, R., Kapfhamer, J., Tsao, F., \& Cushman, F. (2008). Grammatical pattern learning by human infants and cotton-top tamarin monkeys. Cognition, 107, 479-500.

Scott, R., \& Dienes, Z. (2008). The conscious, the unconscious, and familiarity. Journal of Experimental Psychology: Learning Memory, and Cognition, 34, 12641288.

Shanks, D. R., \& St. John, M. F. (1994). Characteristics of dissociable human learning system. Behavioral and Brain Sciences, 17, 367-447.

Tunney, R. J. \& Shanks, D. R. (2003). Does opposition logic provide evidence for conscious and unconscious processes in artificial grammar learning? Consciousness and Cognition, 12, 201-218.

Wang, Q. (2008). L2 stress perception: The reliance on different acoustic cues. Speech Prosody 2008. Campinas, Brazil, 135-138

Warker, J. A., \& Dell, G. S. (2006). Speech errors reflect newly learned phonotactic constraints. Journal of Experimental Psychology: Learning, Memory, and Cognition, 32, 387-398. 